\title{
Disjoint strict singularity of inclusions between rearrangement invariant spaces
}

\author{
by \\ Francisco L. Hernández (Madrid), \\ VíCTOR M. SÁNCHEZ (Madrid), and \\ Evgueni M. Semenov (Voronezh)
}

\begin{abstract}
It is studied when inclusions between rearrangement invariant function spaces on the interval $[0, \infty)$ are disjointly strictly singular operators. In particular suitable criteria, in terms of the fundamental function, for the inclusions $L^{1} \cap L^{\infty} \hookrightarrow E$ and $E \hookrightarrow L^{1}+L^{\infty}$ to be disjointly strictly singular are shown. Applications to the classes of Lorentz and Marcinkiewicz spaces are given.
\end{abstract}

1. Introduction. An operator between two Banach spaces is said to be strictly singular (or Kato) if it fails to be an isomorphism on any infinitedimensional closed subspace. The class of strictly singular operators is a well known closed operator ideal. A weaker notion for Banach lattices is that of disjoint strict singularity: an operator $T$ from a Banach lattice $X$ to a Banach space $Y$ is said to be Disjointly Strictly Singular (DSS for short) if there is no sequence $\left(x_{n}\right)_{n=1}^{\infty}$ of disjointly supported non-null vectors in $X$ such that the restriction of $T$ to the closed subspace spanned by $\left(x_{n}\right)_{n=1}^{\infty}$ is an isomorphism. This notion, introduced in $[\mathrm{HR}]$, is useful in the study of the lattice structure of function spaces (e.g. in constructing function spaces with singular $\ell_{p}$-complemented copies).

The aim of this paper is to study the disjoint strict singularity of the inclusion operator between arbitrary rearrangement invariant spaces (r.i. spaces for short) on the interval $[0, \infty)$.

The analogous problem of DSS inclusions between r.i. spaces on the finite interval $[0,1]$ has been studied by Astashkin ([A]), Novikov $\left(\left[\mathrm{N}_{1}\right],\left[\mathrm{N}_{2}\right]\right)$ and García del Amo, Ruiz and the present authors ([GHSS], [GHR]). If $E$ is

2000 Mathematics Subject Classification: Primary 46E30.

Research of F. L. Hernández and V. M. Sánchez partially supported by DGES (Spain), grant PB97-0240.

Research of E. M. Semenov partially supported by RFFI (Russia), grant 98-01-00044 and by Universities of Russia grant 3667 . 
an r.i. space on $[0,1]$ different from $L^{1}[0,1]$ then the canonical inclusion $E \hookrightarrow L^{1}[0,1]$ is always DSS. This property in fact characterizes $L^{1}[0,1]$ : an r.i. space $F$ such that for any other different r.i. space $E$ with $E \hookrightarrow F$ the inclusion $E \hookrightarrow F$ is DSS must be $L^{1}[0,1]$. A symmetric characterization also holds for $L^{\infty}[0,1]$ and both are deduced from a factorization result given in [GHSS]: for any inclusion $E \hookrightarrow F$ with $E \neq L^{\infty}[0,1]$ and $F \neq L^{1}[0,1]$ there exists an intermediate r.i. space $G$ such that the inclusions $E \hookrightarrow G$ and $G \hookrightarrow F$ are not DSS. In the special context of Orlicz spaces, characterizations of when the inclusion operator $L^{\varphi}(\mu) \hookrightarrow L^{\psi}(\mu)$ is DSS have been given by Kalton $\left[K_{1}\right]$ for sequence spaces with basis (where the notions of disjoint strict singularity and strict singularity coincide) and in [HR] and [GHR] for function spaces. In particular concrete criteria on the functions $\varphi, \psi$ for the inclusions $L^{p}(\mu) \hookrightarrow L^{\psi}(\mu)$ and $L^{\varphi}(\mu) \hookrightarrow L^{p}(\mu)$ to be DSS were given. The classes of Lorentz function spaces $\Lambda(\phi)[0,1]$ and Marcinkiewicz function spaces $M(\phi)[0,1]$ have been studied in [A].

In general it is more delicate to determine the DSS behavior in the $[0, \infty)$ case than in the $[0,1]$ case. Thus, natural r.i. spaces with the same Boyd indices may have different behavior (e.g. the spaces $L^{p} \cap L^{q}$ and $L^{p}+L^{q}$ with respect to the inclusion in $\left.L^{1}+L^{\infty}\right)$. First we analyse in Section 3 the inclusion $L^{1} \cap L^{\infty} \hookrightarrow E$ characterizing the r.i. spaces $E$ in terms of the associated fundamental function $\phi_{E}$. Theorem 3.4 states that $L^{1} \cap L^{\infty} \hookrightarrow E$ is DSS if and only if

$$
\lim _{t \rightarrow 0} \phi_{E}(t)=\lim _{t \rightarrow \infty} \frac{\phi_{E}(t)}{t}=0 .
$$

These conditions are also equivalent to $L^{1} \cap L^{\infty} \hookrightarrow E$ being either strictly singular or weakly compact. In the proof of these statements we make use of the Dunford-Pettis property of $L^{1} \cap L^{\infty}$. This was obtained by Kalton [K $\mathrm{K}_{2}$, during a visit to Madrid in the Spring of 1996, and it has also been proved by Kamińska and Mastyło [KM]. In this section we also determine when the canonical inclusion between a Lorentz space $\Lambda(\phi)$ and the Marcinkiewicz space with the same fundamental function $M(\widetilde{\phi})$ is weakly compact (Proposition 3.1). This extends earlier results for the $[0,1]$ case given by KuzinAleksinskiı̌ [K-A].

In Section 4 we study the disjoint strict singularity of the inclusion $E \hookrightarrow L^{1}+L^{\infty}$ which is in general more complicated to determine; here the functions $t^{-1 / p} \chi_{(0, \infty)}$ play a special role. It is proved that if $E \hookrightarrow L^{1}+L^{\infty}$ is DSS then

$$
\lim _{t \rightarrow 0} \frac{\phi_{E}(t)}{t}=\lim _{t \rightarrow \infty} \phi_{E}(t)=\infty
$$

and

$$
\sup _{n}\left\|t^{-1 / p} \chi_{(1 / n, n)}\right\|_{E}=\infty \quad \text { for any } 1<p<\infty .
$$


In particular the inclusion between the order continuous weak $L^{p}$-space $L_{0}^{p, \infty}$ and $L^{1}+L^{\infty}$ is not DSS. One of the main results of this section (Theorem 4.5) gives a useful sufficient condition to be DSS: if an r.i. space $E$ (different from $L^{1}$ and $L^{\infty}$ ) has submultiplicative fundamental function, then $E \hookrightarrow$ $L^{1}+L^{\infty}$ is DSS, except when $E=L^{p, \infty}$ or $E=L_{0}^{p, \infty}$ for some $1<p<\infty$. This result is obtained by carefully analyzing the inclusion of the associated Marcinkiewicz space $M\left(\widetilde{\phi}_{E}\right)$ in $L^{1}+L^{\infty}$ (Lemma 4.4). As a consequence, we deduce some sufficient conditions on intermediate spaces $F$ between $E$ and $L^{1}+L^{\infty}$ for the inclusion operator $E \hookrightarrow F$ to be DSS (Corollaries 4.6 and 4.7). We also give a criterion for inclusions between Lorentz spaces (Theorem 4.8): If $\lim _{t \rightarrow 0, \infty} \psi(t) / \phi(t)=0$ and $\phi$ is submultiplicative then $\Lambda(\phi) \hookrightarrow \Lambda(\psi)$ is DSS.

2. Notations and previous results. Let us give some definitions and notations. We consider the interval $[0, \infty)$ and the Lebesgue measure $\lambda$. The distribution function $\lambda_{x}$ associated with a measurable function $x$ on $[0, \infty)$ is defined by $\lambda_{x}(s)=\lambda\{t \in[0, \infty):|x(t)|>s\}$, and the decreasing rearrangement function $x^{*}$ of $x$ is

$$
x^{*}(t)=\inf \left\{s \in[0, \infty): \lambda_{x}(s) \leq t\right\} .
$$

A Banach space $E[0, \infty) \equiv E$ of measurable functions defined on $[0, \infty)$ is said to be a rearrangement invariant space (briefly r.i. space) if the following conditions are satisfied:

(a) if $y \in E$ and $|x(t)| \leq|y(t)| \lambda$-a.e. on $[0, \infty)$ then $x \in E$ and $\|x\|_{E} \leq$ $\|y\|_{E}$,

(b) if $y \in E$ and $\lambda_{x}=\lambda_{y}$ then $x \in E$ and $\|x\|_{E}=\|y\|_{E}$.

It is well known that every r.i. space $E$ satisfies the condition $L^{1} \cap L^{\infty} \hookrightarrow$

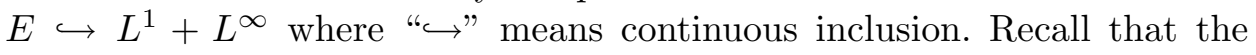
fundamental function $\phi_{E}$ of an r.i. space $E$ is defined by $\phi_{E}(t)=\left\|\chi_{[0, t]}\right\|_{E}$ with $t \geq 0$. It is an increasing function and the associated function $\widetilde{\phi}_{E}$, defined by $\widetilde{\phi}_{E}(t)=t / \phi_{E}(t)$, is also increasing.

The Köthe dual $E^{\prime}$ of an r.i. space $E$ is formed by the measurable functions $x$ on $[0, \infty)$ such that

$$
\|x\|_{E^{\prime}}=\sup _{y \in B_{E}} \int_{0}^{\infty} x(t) y(t) d t<\infty
$$

where $B_{E}$ is the unit ball of $E$. The space $E^{\prime}$ is also an r.i. space and we denote $\left(E^{\prime}\right)^{\prime}$ by $E^{\prime \prime}$. We shall consider r.i. spaces which are either maximal (i.e. $E=E^{\prime \prime}$ ) or minimal (i.e. $E$ is the closed linear span of the simple integrable functions in $\left.E^{\prime \prime}\right)$. 
We consider the Hardy-Littlewood-Pólya semi-order " $\prec$ ": If $x, y \in$ $L^{1}+L^{\infty}$, we say that $x \prec y$ if

$$
\int_{0}^{t} x^{*}(s) d s \leq \int_{0}^{t} y^{*}(s) d s \quad \text { for every } t \in[0, \infty) .
$$

If $E$ is an r.i. space and $x \prec y$ with $y \in E$, then $x \in E$ and $\|x\|_{E} \leq\|y\|_{E}$ (cf. $\left[\mathrm{LT}_{2}\right.$, p. 125]).

An r.i. space $E$ has the Fatou property if for any increasing positive sequence $\left(x_{n}\right)_{n=1}^{\infty}$ in $E$ with $\sup _{n}\left\|x_{n}\right\|_{E}<\infty$ we have $\sup _{n} x_{n} \in E$ and $\left\|\sup _{n} x_{n}\right\|_{E}=\sup _{n}\left\|x_{n}\right\|_{E}$. The Köthe dual $E^{\prime}$ has the Fatou property. Given r.i. spaces $E_{1}$ and $E_{2}$, we consider the sum space $E_{1}+E_{2}$ with the norm $\|x\|_{E_{1}+E_{2}}=\inf \left\{\left\|x_{1}\right\|_{E_{1}}+\left\|x_{2}\right\|_{E_{2}}: x=x_{1}+x_{2}, x_{1} \in E_{1}, x_{2} \in E_{2}\right\}$, and the intersection space $E_{1} \cap E_{2}$ with the norm $\|x\|_{E_{1} \cap E_{2}}=$ $\max \left(\|x\|_{E_{1}},\|x\|_{E_{2}}\right)$. Both are r.i. spaces with fundamental functions $\phi_{E_{1}+E_{2}}$ $=\min \left(\phi_{E_{1}}, \phi_{E_{2}}\right)$ and $\phi_{E_{1} \cap E_{2}}=\max \left(\phi_{E_{1}}, \phi_{E_{2}}\right)$. If $E_{1}=L^{1}$ and $E_{2}=L^{\infty}$ then

$$
\|x\|_{L^{1}+L^{\infty}}=\int_{0}^{1} x^{*}(t) d t=\sup _{\lambda(E)=1} \int_{E}|x(t)| d t .
$$

Important examples of r.i. spaces are the Orlicz, Lorentz and Marcinkiewicz spaces:

If $\varphi$ is a positive convex function on $[0, \infty)$ with $\varphi(0)=0$, the Orlicz space $L^{\varphi}$ consists of all measurable functions $x$ on $[0, \infty)$ for which

$$
\|x\|_{L^{\varphi}}=\inf \left\{s>0: \int_{0}^{\infty} \varphi\left(\frac{|x(t)|}{s}\right) d t \leq 1\right\}<\infty .
$$

If $1<p \leq \infty$ and $1 \leq q \leq \infty$, the classical Lorentz space $L^{p, q}$ consists of all measurable functions $x$ defined on $[0, \infty)$ such that

$$
\|x\|_{p, q}=\left(\int_{0}^{\infty}\left(t^{1 / p} x^{*}(t)\right)^{q} \frac{d t}{t}\right)^{1 / q}<\infty \quad \text { if } q<\infty,
$$

and

$$
\|x\|_{p, \infty}=\sup _{t>0}\left\{t^{1 / p} x^{*}(t)\right\}<\infty .
$$

We shall denote by $L_{0}^{p, \infty}$ the order continuous part of $L^{p, \infty}$.

Let $\Phi$ be the class of all increasing concave functions $\phi$ on $[0, \infty)$ with $\phi(0)=0$. If $\phi \in \Phi$ the Lorentz space $\Lambda(\phi)$ consists of all measurable functions $x$ defined on $[0, \infty)$ such that

$$
\|x\|_{\Lambda(\phi)}=\int_{0}^{\infty} x^{*}(t) d \phi(t)<\infty .
$$


The Marcinkiewicz space $M(\phi)$ consists of all measurable functions $x$ defined on $[0, \infty)$ for which

$$
\|x\|_{M(\phi)}=\sup _{t>0} \frac{\int_{0}^{t} x^{*}(s) d s}{\phi(t)}<\infty .
$$

Given $\phi$, the spaces $\Lambda(\phi)$ and $M(\widetilde{\phi})$ are respectively the smallest and the biggest r.i. space having the same fundamental function $\phi$ (cf. [KPS, Theorems 5.5 and 5.7]]):

THEOREM 2.1. Every r.i. space $E$ with fundamental function $\phi$ satisfies

$$
\Lambda(\phi) \hookrightarrow E \hookrightarrow M(\widetilde{\phi}) .
$$

Given $\phi \in \Phi$, we will consider the subspace $M_{0}(\phi)$ of $M(\phi)$ consisting of all functions $x \in M(\phi)$ such that

$$
\lim _{t \rightarrow 0, \infty} \frac{1}{\phi(t)} \int_{0}^{t} x^{*}(s) d s=0 .
$$

It is clear that if $M_{0}(\phi) \neq\{0\}$, then the function $\phi$ must satisfy

$$
\lim _{t \rightarrow 0} \frac{\phi(t)}{t}=\lim _{t \rightarrow \infty} \phi(t)=\infty .
$$

Conversely, it is well known that under both conditions and

$$
\lim _{t \rightarrow 0} \phi(t)=0
$$

the space $M_{0}(\phi)$ is a separable closed subspace of $M(\phi)$ and $\left(M_{0}(\phi)\right)^{*}=$ $\Lambda(\phi)$ (cf. [KPS, Theorem II.5.4]). The function $\phi$ is the fundamental function of the space $M_{0}(\widetilde{\phi})$. Recall also that if $\lim _{t \rightarrow 0} \phi(t)=0$ and $\lim _{t \rightarrow \infty} \phi(t)=\infty$ then the Lorentz space $\Lambda(\phi)$ is separable and $(\Lambda(\phi))^{*}=M(\phi)$. In general given an increasing function $\phi$, since the function $\widetilde{\phi}$ is quasiconcave, there exists a concave function $\bar{\phi}$ such that $\widetilde{\phi} \leq \bar{\phi} \leq 2 \widetilde{\phi}$ (cf. [BS], [KPS]).

For other properties of r.i. spaces we refer to [BS], [KPS], [LT $\left.{ }_{2}\right]$.

3. The inclusion $L^{1} \cap L^{\infty} \hookrightarrow E$. In this section we characterize when the inclusion $L^{1} \cap L^{\infty} \hookrightarrow E$ is DSS. We will use the following

Proposition 3.1. Let $\phi \in \Phi$. The inclusion operator $\Lambda(\phi) \hookrightarrow M(\widetilde{\phi})$ is weakly compact if and only if the following conditions hold:

$$
\lim _{t \rightarrow 0} \frac{\phi(t)}{t}=\lim _{t \rightarrow \infty} \phi(t)=\infty
$$

and

$$
\lim _{t \rightarrow 0} \phi(t)=\lim _{t \rightarrow \infty} \frac{\phi(t)}{t}=0 .
$$


Proof. First assume that (1) and (2) hold. If $j$ denotes the inclusion operator, we have, by Theorem 2.1,j: $\Lambda(\phi) \hookrightarrow M_{0}(\widetilde{\phi}) \hookrightarrow M(\widetilde{\phi})$. Hence the adjoint operator $j^{*}$ acts from $\left(M_{0}(\bar{\phi})\right)^{*}=\Lambda(\bar{\phi})$ into $(\Lambda(\phi))^{*}$ and then $j^{* *}$ transforms $(\Lambda(\phi))^{* *}$ into $M(\bar{\phi})$. Thus, by a theorem of Gantmacher (see e.g. [PR, p. 250]) we conclude that $j$ is weakly compact.

Conversely, if any of the conditions in (1) or (2) fails, then the inclusion operator $\Lambda(\phi) \hookrightarrow M(\widetilde{\phi})$ is not weakly compact since then it cannot be factorized through any reflexive space. Indeed, consider the space $H$ of all functions $x(t)=\sum_{k} x_{k} \chi_{[k-1, k)}$ with $x_{k} \rightarrow 0$. It is easy to check that if $\lim _{t \rightarrow \infty} \phi(t)<\infty$ then

$$
\|x\|_{\Lambda(\phi)} \approx\|x\|_{M(\widetilde{\phi})} \approx \sup _{k}\left|x_{k}\right|
$$

and if $\lim _{t \rightarrow \infty} \phi(t) / t>0$ then

on the subspace $H$.

$$
\|x\|_{\Lambda(\phi)} \approx\|x\|_{M(\widetilde{\phi})} \approx \sum_{k}\left|x_{k}\right|
$$

Consider now the space $V$ of all functions $x \in L^{\infty}$ with $\operatorname{supp}(x) \subset[0,1]$. On $V$, if $\lim _{t \rightarrow 0} \phi(t)>0$ then

$$
\|x\|_{\Lambda(\phi)} \approx\|x\|_{M(\widetilde{\phi})} \approx\|x\|_{L^{\infty}},
$$

and if $\lim _{t \rightarrow 0} \phi(t) / t<\infty$ then

$$
\|x\|_{\Lambda(\phi)} \approx\|x\|_{M(\tilde{\phi})} \approx\|x\|_{L^{1}}
$$

Proposition 3.2. Given $\phi \in \Phi$, there exists a reflexive r.i. space $E$ with fundamental function $\phi_{E}=\phi$ if and only if the function $\phi$ satisfies conditions (1) and (2).

Proof. The necessity part follows directly from Proposition 3.1 and Theorem 2.1. Conversely, under conditions (1) and (2), the inclusion operator $\Lambda(\phi) \hookrightarrow M(\widetilde{\phi})$ is weakly compact. Consider the real interpolation space $E=(\Lambda(\phi), M(\widetilde{\phi}))_{\theta, p}$ for $0<\theta<1$ and $1<p<\infty$. Then, using [B, Proposition II.3.1] we deduce that $E$ is a reflexive r.i. space, and it is clear that its fundamental function $\phi_{E}$ is equal to $\phi$.

The above statements extend previous results for r.i. spaces on $[0,1]$ given by Kuzin-Aleksinskiü [K-A].

The following lemma will also be useful in order to characterize when the inclusion operator $L^{1} \cap L^{\infty} \hookrightarrow E$ is DSS.

LEMma 3.3. If $\phi \in \Phi$ satisfies condition (2) then there exists a function $\psi \in \Phi$ such that $\psi$ satisfies conditions (1) and (2), and

$$
\int_{0}^{\infty} \phi^{\prime}(t) \psi^{\prime}(t) d t<\infty .
$$


Proof. This can be deduced from [P, Theorem 5]. We also give a selfcontained alternative short proof provided by the referee. Using condition (2) we have

$$
\lim _{t \rightarrow \infty} \phi^{\prime}(t) \leq \lim _{t \rightarrow \infty} \frac{1}{t} \int_{0}^{t} \phi^{\prime}(s) d s=\lim _{t \rightarrow \infty} \frac{\phi(t)}{t}=0 .
$$

Choose a strictly increasing unbounded sequence $\left(a_{n}\right)_{n=1}^{\infty}$ which satisfies $a_{1}=1$,

$$
a_{n+1} \geq 2 a_{n}-a_{n-1} \quad \text { and } \quad \phi^{\prime}\left(a_{n}\right) \leq 1 / n .
$$

Also, choose a strictly decreasing sequence $\left(b_{n}\right)_{n=0}^{\infty}$ in $(0,1]$ such that

$$
\phi\left(b_{n}\right)=2^{-n} \phi(1)
$$

(so in particular $\left.b_{0}=1\right)$. Now we consider the function $f:(0, \infty) \rightarrow(0, \infty)$ defined by

$$
f=\sum_{n=0}^{\infty}(n+1) \chi_{\left(b_{n+1}, b_{n}\right]}+\sum_{n=1}^{\infty} \frac{1}{n\left(a_{n+1}-a_{n}\right)} \chi_{\left(a_{n}, a_{n+1}\right]}
$$

and the function

$$
\psi(t)=\int_{0}^{t} f(s) d s .
$$

It is easy to verify that $\psi$ has all the required properties.

Theorem 3.4. Let $E$ be an r.i. space. The following conditions are equivalent:

(i) The inclusion operator $L^{1} \cap L^{\infty} \hookrightarrow E$ is DSS.

(ii) The inclusion operator $L^{1} \cap L^{\infty} \hookrightarrow E$ is strictly singular.

(iii) The inclusion operator $L^{1} \cap L^{\infty} \hookrightarrow E$ is weakly compact.

(iv) $\lim _{t \rightarrow 0} \phi_{E}(t)=\lim _{t \rightarrow \infty} \phi_{E}(t) / t=0$.

Proof. (i) $\Rightarrow$ (iv). Suppose that $\lim _{t \rightarrow 0} \phi_{E}(t)=c>0$. Then

$$
\|x\|_{E} \geq \lim _{t \rightarrow 0}\left\|x^{*} \chi_{[0, t]}\right\|_{E} \geq \lim _{t \rightarrow 0} x^{*}(t) \phi_{E}(t)=c\|x\|_{\infty}
$$

for every $x \in E$. If $V$ is the subspace of functions $x \in L^{\infty}$ with $\operatorname{supp}(x) \subset$ $[0,1]$ then $\|x\|_{1} \leq\|x\|_{\infty}$ for $x \in V$. Hence $\|x\|_{L^{1} \cap L^{\infty}}=\|x\|_{\infty}$. Thus $\|x\|_{L^{1} \cap L^{\infty}} \leq c^{-1}\|x\|_{E}$ for $x \in V$. Since the converse inequality always holds, we deduce that the norms $\|\cdot\|_{L^{1} \cap L^{\infty}}$ and $\|\cdot\|_{E}$ are equivalent on $V$, so the inclusion $L^{1} \cap L^{\infty} \hookrightarrow E$ is not DSS.

Suppose now that $\lim _{t \rightarrow \infty} \phi_{E}(t) / t=b>0$. Then $\phi_{E}(t) \geq b t$ for every $t>0$. Hence $\widetilde{\phi}_{E}(t) \leq 1 / b$. Therefore, using Theorem 2.1, we have

$$
\|x\|_{E} \geq\|x\|_{M\left(\widetilde{\phi}_{E}\right)} \geq b\|x\|_{L^{1}}
$$


for every $x \in E$. Now, if $H$ is the subspace of functions $x=\sum_{k} x_{k} \chi_{[k-1, k)}$, then

$$
\|x\|_{E} \geq b \sum_{k}\left|x_{k}\right|
$$

for every $x \in H$. Hence

$$
\|x\|_{L^{1} \cap L^{\infty}}=\sum_{k}\left|x_{k}\right| \leq \frac{1}{b}\|x\|_{E}
$$

for every $x \in H$. As the converse inequality also holds, we conclude that the inclusion $L^{1} \cap L^{\infty} \hookrightarrow E$ is not DSS.

(iv) $\Rightarrow$ (iii). Let $\bar{\phi}_{E}$ be the least concave majorant function of $\phi_{E}$. Then, by Lemma 3.3 , there exists $\psi \in \Phi$ which satisfies (1) and (2) and

$$
\int_{0}^{\infty} \bar{\phi}_{E}^{\prime}(t) \psi^{\prime}(t) d t<\infty .
$$

From (3) we deduce the inclusion $M(\psi) \hookrightarrow \Lambda\left(\bar{\phi}_{E}\right)$. Now, if we denote by $\bar{\psi}$ the least concave majorant function of $\widetilde{\psi}$, then $\Lambda(\bar{\psi}) \hookrightarrow M(\psi)$. Thus, we have got the factorization

$$
L^{1} \cap L^{\infty} \hookrightarrow \Lambda(\bar{\psi}) \hookrightarrow M(\psi) \hookrightarrow \Lambda\left(\bar{\phi}_{E}\right) \hookrightarrow E .
$$

Now, by Proposition 3.1, the inclusion $\Lambda(\bar{\psi}) \hookrightarrow M(\psi)$, and hence $L^{1} \cap$ $L^{\infty} \hookrightarrow E$, is weakly compact.

(iii) $\Rightarrow$ (ii) follows from the Dunford-Pettis property of $L^{1} \cap L^{\infty}\left(\left[\mathrm{K}_{2}\right]\right.$, $[\mathrm{KM}]$ ), and (ii) $\Rightarrow(\mathrm{i})$ is trivial.

REMARK. A characterization of when the inclusion $L^{1} \cap L^{\infty} \hookrightarrow E$ is strictly singular has also been obtained very recently by Cobos, Manzano, Martínez and Matos in [CMMM, Theorem 3.4] using a different technique based on qualitative interpolation methods.

Reasoning as in the above implication (iv) $\Rightarrow$ (iii) we get the following

Corollary 3.5. Given an r.i. space E, there exists a reflexive r.i. space $F$ such that $F \hookrightarrow E$ if and only if the condition (iv) is satisfied.

Using duality arguments and the equality $\phi_{E}(t) \phi_{E^{\prime}}(t)=t$ we also deduce the following

Corollary 3.6. Given an r.i. space E, there exists a reflexive r.i. space $F$ such that $E \hookrightarrow F$ if and only if the condition (1) is satisfied.

The above corollaries for r.i. spaces on $[0,1]$ were given in $[\mathrm{K}-\mathrm{A}]$ and $\left[\mathrm{N}_{1}\right]$.

4. The inclusion $E \hookrightarrow L^{1}+L^{\infty}$. In this section we study when the inclusion $E \hookrightarrow L^{1}+L^{\infty}$ is DSS. First we give some necessary conditions. 
THEOREM 4.1. Let $E$ be an r.i. space. If the inclusion operator $E \hookrightarrow$ $L^{1}+L^{\infty}$ is DSS, then:

(i) $\lim _{t \rightarrow 0} \phi_{E}(t) / t=\lim _{t \rightarrow \infty} \phi_{E}(t)=\infty$.

(ii) $t^{-1 / p} \chi_{(0, \infty)} \notin E$ for any $1<p<\infty$.

Proof. (i) First suppose that $\lim _{t \rightarrow 0} \phi_{E}(t) / t<\infty$. If we consider the sequence $\left(x_{n}\right)_{n=1}^{\infty}=\left(2^{n} \chi_{\left(2^{-n}, 2^{-n+1}\right]}\right)_{n=1}^{\infty}$, the norms of $E$ and $L^{1}+L^{\infty}$ are equivalent on the subspace $\left[\left(x_{n}\right)_{n=1}^{\infty}\right]$, which is isomorphic to $\ell_{1}$. Indeed, since $\left\|x_{n}\right\|_{E}=2^{n} \phi_{E}\left(2^{-n}\right) \leq M$ for some $M<\infty$ and every $n \in \mathbb{N}$, we have

$$
\sum_{n}\left|a_{n}\right|=\left\|\sum_{n} a_{n} x_{n}\right\|_{L^{1}+L^{\infty}} \leq\left\|\sum_{n} a_{n} x_{n}\right\|_{E} \leq M \sum_{n}\left|a_{n}\right|
$$

for every scalar sequence $\left(a_{n}\right)_{n=1}^{\infty}$.

If $\lim _{t \rightarrow \infty} \phi_{E}(t)=A<\infty$, then the sequence $\left(\chi_{[n-1, n)}\right)_{n=1}^{\infty}$ in $E$ and $L^{1}+L^{\infty}$ is equivalent to the canonical basis of $c_{0}$ since

$$
\sup _{n}\left|a_{n}\right|=\left\|\sum_{n} a_{n} \chi_{[n-1, n)}\right\|_{L^{1}+L^{\infty}} \leq\left\|\sum_{n} a_{n} \chi_{[n-1, n)}\right\|_{E} \leq A \sup _{n}\left|a_{n}\right|
$$

for every scalar sequence $\left(a_{n}\right)_{n=1}^{\infty}$.

(ii) Suppose now that there exists $1<p<\infty$ such that $t^{-1 / p} \chi_{(0, \infty)} \in E$. Let $\left(x_{n}\right)_{n=1}^{\infty}$ be a sequence of disjointly supported functions in $E$ such that $x_{n}$ and $t^{-1 / p} \chi_{(0, \infty)}$ are equimeasurable for every $n \in \mathbb{N}$. Then the functions $\sum_{n} a_{n} x_{n}$ and $\|a\|_{p} t^{-1 / p} \chi_{(0, \infty)}$ are equimeasurable for every scalar sequence $a=\left(a_{n}\right)_{n=1}^{\infty}$. Therefore

$$
\begin{aligned}
\left\|t^{-1 / p} \chi_{(0, \infty)}\right\|_{L^{1}+L^{\infty}}\|a\|_{p} & =\left\|\sum_{n} a_{n} x_{n}\right\|_{L^{1}+L^{\infty}} \leq\left\|\sum_{n} a_{n} x_{n}\right\|_{E} \\
& =\left\|t^{-1 / p} \chi_{(0, \infty)}\right\|_{E}\|a\|_{p}
\end{aligned}
$$

for every scalar sequence $a=\left(a_{n}\right)_{n=1}^{\infty}$. So $\left(x_{n}\right)_{n=1}^{\infty}$ is equivalent in $E$ and $L^{1}+L^{\infty}$ to the canonical basis of $\ell_{p}$.

From the above theorem we see that the inclusion $L^{p, \infty} \hookrightarrow L^{1}+L^{\infty}$ is not DSS for any $1<p<\infty$.

We turn to showing that the above conditions (i) and (ii) are not in general sufficient for $E \hookrightarrow L^{1}+L^{\infty}$ to be DSS:

Proposition 4.2. Let $1<p<\infty$ and $\left(x_{n}\right)_{n=1}^{\infty}$ be a disjointly supported sequence of functions in $L^{p, \infty}$ with $\left\|x_{n}\right\|_{p, \infty} \leq 1$ for every $n \in \mathbb{N}$. Let $\left(\varepsilon_{n}\right)_{n=1}^{\infty}$ be a sequence in $[0,1]$ with $\sum_{n} \varepsilon_{n}<\infty$ and $c$ be a positive constant such that

$$
\int_{0}^{\tau} x_{n}^{*}(t) d t \geq c \tau^{1-1 / p}
$$


for every $n \in \mathbb{N}$ and every $\tau \in\left[\varepsilon_{n}, 1\right]$. Then there exists a constant $M>0$ such that

$$
\frac{1}{M}\|a\|_{p} \leq\left\|\sum_{n} a_{n} x_{n}\right\|_{L^{1}+L^{\infty}} \leq\left\|\sum_{n} a_{n} x_{n}\right\|_{p, \infty} \leq M\|a\|_{p}
$$

for every $a=\left(a_{n}\right)_{n=1}^{\infty} \in \ell_{p}$.

Proof. Since $\|x\|_{L^{1}+L^{\infty}}=\sup _{\lambda(E)=1} \int_{E}|x(t)| d t$, we have

$$
\left\|\sum_{n} a_{n} x_{n}\right\|_{L^{1}+L^{\infty}}=\sup _{\sum_{n} \tau_{n}=1} \sum_{n}\left|a_{n}\right| \int_{0}^{\tau_{n}} x_{n}^{*}(t) d t .
$$

Choose $n_{0} \in \mathbb{N}$ such that $\sum_{n=n_{0}}^{\infty} \varepsilon_{n} \leq 1 / 2$. Then, by (4), we have

$$
\begin{aligned}
\left\|\sum_{n} a_{n} x_{n}\right\|_{L^{1}+L^{\infty}} & \geq c \sup _{\substack{\tau_{n} \geq \varepsilon_{n} \\
\sum_{n=n_{0}}^{\infty} \tau_{n}=1}} \sum_{n=n_{0}}^{\infty}\left|a_{n}\right| \tau_{n}^{1-1 / p} \\
& \geq c \sup _{\substack{\sum_{n=n_{0}}^{\infty} \tau_{n} \geq 0 \\
\tau_{n}=1 / 2}} \sum_{n=n_{0}}^{\infty}\left|a_{n}\right| \tau_{n}^{1-1 / p} \\
& =2^{1 / p-1} c \sup _{\substack{\sum_{n=n_{0}}^{\infty} \tau_{n}=1 \\
\tau_{n}}} \sum_{n=n_{0}}^{\infty}\left|a_{n}\right| \tau_{n}^{1-1 / p} \\
& =2^{1 / p-1} c\left(\sum_{n=n_{0}}^{\infty}\left|a_{n}\right|^{p}\right)^{1 / p} .
\end{aligned}
$$

As $\left\|\sum_{n} a_{n} x_{n}\right\|_{L^{1}+L^{\infty}} \geq c \sup _{n}\left|a_{n}\right|$ we obtain the first inequality of (5).

The second inequality of (5) is obvious. Finally, since $L^{p, \infty}$ satisfies an upper $p$-estimate (cf. $\left[\mathrm{CD}_{1}\right]$ ), we obtain the third inequality of (5).

Corollary 4.3. The inclusion $L_{0}^{p, \infty} \hookrightarrow L^{1}+L^{\infty}$ is not DSS for any $1<p<\infty$.

Proof. For a sequence $\left(\varepsilon_{n}\right)_{n=1}^{\infty}$ as in Proposition 4.2 we just consider a disjointly supported sequence of functions $\left(x_{n}\right)_{n=1}^{\infty}$ in $L_{0}^{p, \infty}$ such that

$$
x_{n}^{*}(t)= \begin{cases}\varepsilon_{n}^{-1 / p} & \text { if } 0 \leq t \leq \varepsilon_{n} \\ t^{-1 / p} & \text { if } \varepsilon_{n}<t<1 \\ 0 & \text { if } t \geq 1\end{cases}
$$

REMARK. The above corollary shows that in general the conditions (i) and (ii) of Theorem 4.1 are not sufficient for $E \hookrightarrow L^{1}+L^{\infty}$ to be DSS. It also shows that the condition (ii) can be replaced, using the Fatou property of $E^{\prime \prime}$, by the sharper one:

$$
\sup _{n}\left\|t^{-1 / p} \chi_{(1 / n, n)}\right\|_{E}=\infty \quad \text { for any } 1<p<\infty .
$$


We shall give some sufficient conditions for $E \hookrightarrow L^{1}+L^{\infty}$ to be DSS. A very strong sufficient condition is that $1 / \phi_{E} \in L^{p}$ for some $1<p<\infty$ since in this case we have the inclusion $M\left(\widetilde{\phi}_{E}\right) \hookrightarrow L^{p}$. One of the main results of this section (Theorem 4.5) gives a milder sufficient condition for $E \hookrightarrow L^{1}+L^{\infty}$ to be DSS. First we analyze the case of Marcinkiewicz spaces.

Let $\phi \in \Phi$ and assume that $M(\phi) \hookrightarrow L^{1}+L^{\infty}$ is not DSS. Then there exist a disjointly supported sequence of functions $\left(x_{n}\right)_{n=1}^{\infty} \subset M(\phi)$ and a constant $D>0$ such that

$$
\left\|\sum_{n} a_{n} x_{n}\right\|_{M(\phi)} \leq D\left\|\sum_{n} a_{n} x_{n}\right\|_{L^{1}+L^{\infty}}
$$

for every scalar sequence $\left(a_{n}\right)_{n=1}^{\infty}$. Define

$$
\varphi_{n}(\tau)=\int_{0}^{\tau} x_{n}^{*}(t) d t
$$

for $n \in \mathbb{N}$ and $\tau>0$. We may assume without loss of generality that $\varphi_{n}(1)=1$ for every $n \in \mathbb{N}$. Under this hypothesis we have the following

Lemma 4.4. There exists an increasing sequence $\left(n_{k}\right)_{k=1}^{\infty}$ of integers such that

$$
\varphi_{n}\left(\frac{1}{k}\right) \geq \frac{1}{D \phi(k)}
$$

for every $k \in \mathbb{N}$ and every $n \geq n_{k}$, where $D$ is the constant in (6).

Proof. The inequality (6) implies

$$
\sup _{\tau_{n} \geq 0} \frac{\sum_{n}\left|a_{n}\right| \int_{0}^{\tau_{n}} x_{n}^{*}(t) d t}{\phi\left(\sum_{n} \tau_{n}\right)} \leq D \sup _{\substack{\tau_{n} \geq 0 \\ \sum_{n} \tau_{n}=1}} \sum_{n}\left|a_{n}\right| \int_{0}^{\tau_{n}} x_{n}^{*}(t) d t
$$

i.e.

$$
\sup _{\tau_{n} \geq 0} \frac{\sum_{n}\left|a_{n}\right| \varphi_{n}\left(\tau_{n}\right)}{\phi\left(\sum_{n} \tau_{n}\right)} \leq D \sup _{\substack{\tau_{n} \geq 0 \\ \sum_{n} \tau_{n}=1}} \sum_{n}\left|a_{n}\right| \varphi_{n}\left(\tau_{n}\right)
$$

or

$$
\sup _{s_{n} \geq 0} \frac{\sum_{n}\left|a_{n}\right| s_{n}}{\phi\left(\sum_{n} \varphi_{n}^{-1}\left(s_{n}\right)\right)} \leq D \sup _{\substack{s_{n} \geq 0 \\ \sum_{n} \varphi_{n}^{-1}\left(s_{n}\right)=1}} \sum_{n}\left|a_{n}\right| s_{n} .
$$

Now, each $\varphi_{n}$ is concave, therefore $\varphi_{n}^{-1}$ is convex and the set

$$
A=\left\{\left(t_{n}\right)_{n=1}^{\infty} \subset \mathbb{R}: \sum_{n} \varphi_{n}^{-1}\left(\left|t_{n}\right|\right) \leq 1\right\}
$$

is convex. Denote by $\ell_{M}$ the modular (or Musielak-Orlicz) sequence space generated by the sequence $\left(\varphi_{n}^{-1}\right)_{n=1}^{\infty}$ (cf. [W], $\left.\left[\mathrm{LT}_{1}\right]\right)$. Then $A$ is the unit ball 
of $\ell_{M}$. The inequality (7) implies that

$$
\sup _{s_{n} \geq 0} \frac{\sum_{n}\left|a_{n}\right| s_{n}}{\phi\left(\sum_{n} \varphi_{n}^{-1}\left(s_{n}\right)\right)} \leq D\|a\|_{\ell_{M}^{\prime}} .
$$

Therefore

$$
\sup _{\|a\|_{\ell_{M}^{\prime}} \leq 1} \sum_{n}\left|a_{n}\right| \frac{s_{n}}{\phi\left(\sum_{m} \varphi_{m}^{-1}\left(s_{m}\right)\right)} \leq D
$$

for each $\left(s_{n}\right)_{n=1}^{\infty}$ with $s_{n} \geq 0$ for every $n \in \mathbb{N}$. Since $\ell_{M}$ is maximal we obtain

$$
\left\|\left(\frac{s_{n}}{\phi\left(\sum_{m} \varphi_{m}^{-1}\left(s_{m}\right)\right)}\right)_{n=1}^{\infty}\right\|_{\ell_{M}} \leq D
$$

and then

$$
\left(\frac{s_{n}}{D \phi\left(\sum_{m} \varphi_{m}^{-1}\left(s_{m}\right)\right)}\right)_{n=1}^{\infty} \in A .
$$

Hence

$$
\sum_{n} \varphi_{n}^{-1}\left(\frac{s_{n}}{D \phi\left(\sum_{m} \varphi_{m}^{-1}\left(s_{m}\right)\right)}\right) \leq 1 .
$$

Given $k \in \mathbb{N}$ and a set $I \subset \mathbb{N}$ with $|I|=k$, we consider the sequence $\left(s_{n}\right)_{n=1}^{\infty}$ defined by

$$
s_{n}= \begin{cases}1 & \text { if } n \in I \\ 0 & \text { if } n \notin I\end{cases}
$$

Using the assumption $\varphi_{n}(1)=1$ for every $n \in \mathbb{N}$ we get

$$
\sum_{n \in I} \varphi_{n}^{-1}\left(\frac{1}{D \phi(k)}\right) \leq 1 .
$$

This means that

$$
\left|\left\{n \in \mathbb{N}: \varphi_{n}^{-1}\left(\frac{1}{D \phi(k)}\right)>\frac{1}{k}\right\}\right|<k
$$

Consequently, there exists a sequence $\left(n_{k}\right)_{k=1}^{\infty}$ as in the statement.

Now we are able to formulate a converse of Proposition 4.2.

Theorem 4.5. Let $E$ be an r.i. space, different from $L^{1}$ and $L^{\infty}$, with submultiplicative fundamental function. If the inclusion operator $E \hookrightarrow$ $L^{1}+L^{\infty}$ is not DSS, then $E=L^{p, \infty}$ or $E=L_{0}^{p, \infty}$ for some $1<p<\infty$.

Proof. There exist a sequence of disjointly supported functions $\left(x_{n}\right)_{n=1}^{\infty}$ $\subset E$ and a constant $D>0$ such that

$$
\left\|\sum_{n} a_{n} x_{n}\right\|_{E} \leq D\left\|\sum_{n} a_{n} x_{n}\right\|_{L^{1}+L^{\infty}}
$$


for every scalar sequence $\left(a_{n}\right)_{n=1}^{\infty}$. Now, by Theorem 2.1, we have

$$
\left\|\sum_{n} a_{n} x_{n}\right\|_{M\left(\widetilde{\phi}_{E}\right)} \leq D\left\|\sum_{n} a_{n} x_{n}\right\|_{L^{1}+L^{\infty}} .
$$

We can assume that $\left(x_{n}\right)_{n=1}^{\infty}$ is normalized in $L^{1}+L^{\infty}$ and $\widetilde{\phi}_{E}$ is concave. Using Lemma 4.4 and the concavity of $\widetilde{\phi}_{E}$ we get

$$
\varphi_{n}(\tau) \geq \frac{1}{2 D \widetilde{\phi}_{E}(1 / \tau)}
$$

for every $k \in \mathbb{N}, \tau \in[1 / k, 1]$ and $n \geq n_{k}$. Indeed, suppose that $\varphi_{n}(\tau)<$ $1 /\left(2 D \widetilde{\phi}_{E}(1 / \tau)\right)$ for some $k \in \mathbb{N}, \tau \in[1 / k, 1]$ and $n \geq n_{k}$. Then there exists $m \in \mathbb{N}$ such that $\tau \in\left(1 / 2^{m}, 1 / 2^{m-1}\right]$. If $1 / k \leq 1 / 2^{m}$ then

$$
\varphi_{n}\left(1 / 2^{m}\right) \leq \frac{1}{2 D \widetilde{\phi}_{E}\left(2^{m-1}\right)} \leq \frac{1}{D \widetilde{\phi}_{E}\left(2^{m}\right)},
$$

which contradicts Lemma 4.4. The other case is analogous.

Now, by the submultiplicativity of $\phi_{E}$ there exists $C>0$ such that

$$
\widetilde{\phi}_{E}(t s) \geq \frac{1}{C} \widetilde{\phi}_{E}(t) \widetilde{\phi}_{E}(s)
$$

for every $t, s>0$. Hence $\widetilde{\phi}_{E}(1 / \tau) \leq C \widetilde{\phi}_{E}(1) / \widetilde{\phi}_{E}(\tau)$ for every $\tau>0$ and

$$
\varphi_{n}(\tau) \geq \frac{1}{2 C D \widetilde{\phi}_{E}(1)} \widetilde{\phi}_{E}(\tau)
$$

for every $k \in \mathbb{N}, \tau \in[1 / k, 1]$ and $n \geq n_{k}$. Therefore, given $j \in \mathbb{N}$, we have

$$
\begin{aligned}
& \sup _{|I|=j}\left\|\sum_{n \in I} x_{n}\right\|_{E} \geq \sup _{|I|=j}\left\|\sum_{n \in I} x_{n}\right\|_{M\left(\widetilde{\phi}_{E}\right)} \geq \sup _{|I|=j} \sup _{0<\tau \leq j} \frac{\sum_{n \in I} \varphi_{n}(\tau / j)}{\widetilde{\phi}_{E}(\tau)}
\end{aligned}
$$

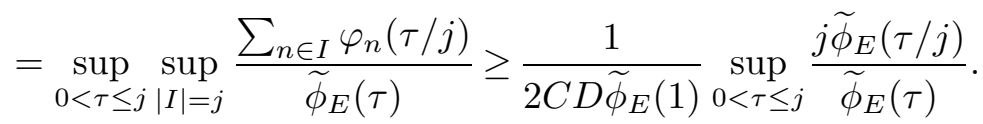

Now, since $\left\|x_{n}\right\|_{M\left(\widetilde{\phi}_{E}\right)} \leq D$ for every $n \in \mathbb{N}$, we have $\varphi_{n}(\tau) \leq D \widetilde{\phi}_{E}(\tau)$ for every $n \in \mathbb{N}$ and $\tau>0$. If $|I|=j$ then

$$
\begin{aligned}
\left\|\sum_{n \in I} x_{n}\right\|_{L^{1}+L^{\infty}} & =\sup _{\sum_{n \in I} \tau_{n}=1} \sum_{n \in I} \varphi_{n}\left(\tau_{n}\right) \\
& \leq \sup _{\sum_{n \in I} \tau_{n}=1} \sum_{n \in I} D \widetilde{\phi}_{E}\left(\tau_{n}\right)=D j \widetilde{\phi}_{E}\left(\frac{1}{j}\right) .
\end{aligned}
$$

Comparing the last two inequalities we get

$$
\frac{1}{2 C D \widetilde{\phi}_{E}(1)} \sup _{0<\tau \leq j} \frac{\widetilde{\phi}_{E}(\tau / j)}{\widetilde{\phi}_{E}(\tau)} \leq D \widetilde{\phi}_{E}\left(\frac{1}{j}\right)
$$


for every $j \in \mathbb{N}$. Consequently,

$$
\widetilde{\phi}_{E}(t s) \leq C_{1} \widetilde{\phi}_{E}(t) \widetilde{\phi}_{E}(s)
$$

for every $t, s>0$ such that $t s \leq 1$ where $C_{1}=4 C D^{2} \widetilde{\phi}_{E}(1)$.

Therefore

$$
\frac{1}{C} \widetilde{\phi}_{E}(t) \widetilde{\phi_{E}}(s) \leq \widetilde{\phi}_{E}(t s) \leq C_{1} \widetilde{\phi}_{E}(t) \widetilde{\phi}_{E}(s)
$$

for every $0<t, s \leq 1$. Now, using the well known fact that the only functions which satisfy such an equivalence are those which are equivalent to powers, and that $\widetilde{\phi}_{E}$ is an increasing concave function on $[0,1]$, we deduce that there exist $C_{2}>1$ and $\alpha \in[0,1]$ such that

$$
\frac{1}{C_{2}} t^{\alpha} \leq \widetilde{\phi}_{E}(t) \leq C_{2} t^{\alpha}
$$

for every $t \in[0,1]$. If $t>1$ and $0<s \leq 1 / t$, then

$$
\frac{\widetilde{\phi}_{E}(t s)}{C_{1} \widetilde{\phi}_{E}(s)} \leq \widetilde{\phi}_{E}(t) \leq C \frac{\widetilde{\phi}_{E}(t s)}{\widetilde{\phi}_{E}(s)}
$$

and

$$
\frac{1}{C_{1} C_{2}^{2}} t^{\alpha} \leq \widetilde{\phi}_{E}(t) \leq C C_{2}^{2} t^{\alpha}
$$

for every $t>0$. If $\alpha=0$ then $E=L^{1}$ and if $\alpha=1$ then $E=L^{\infty}$, which are excluded. Now, there exist $1<p<\infty$ and $C_{3}>1$ such that

$$
\frac{1}{C_{3}} t^{1 / p} \leq \phi_{E}(t) \leq C_{3} t^{1 / p}
$$

for every $t>0$. The right inequality and Theorem 2.1 imply that $E \hookrightarrow L^{p, \infty}$.

Let us now study the converse inclusion. By (8), there exist a constant $C_{4}>0$ and a sequence $\left(r_{n}\right)_{n=1}^{\infty}$ such that

$$
\varphi_{r_{n}}(\tau) \geq C_{4} \tau^{1-1 / p}
$$

for every $n \in \mathbb{N}$ and $\tau \in\left[1 / 2^{n}, 1\right]$. This means that

$$
C_{4}(1-1 / p) \min \left(t^{-1 / p}, 2^{n / p}\right) \chi_{[0,1]} \prec x_{r_{n}}
$$

for every $n \in \mathbb{N}$. Since the functions $\left(x_{n}\right)_{n=1}^{\infty}$ are disjointly supported, for every $\varepsilon>0$ and $j \in \mathbb{N}$ there exist $I \subset \mathbb{N}$ with $|I|=j$ such that

$$
C_{4}(1-1 / p) \sum_{i=1}^{j}(t-i+1)^{-1 / p} \chi_{[i-1+\varepsilon, i]}(t) \prec \sum_{i \in I} x_{i} .
$$

Applying now $\left[\mathrm{LT}_{2}\right.$, Proposition 2.a.8] and Proposition 4.2 we get

$$
C_{4}(1-1 / p)\left\|\sum_{i=1}^{j}(t-i+1)^{-1 / p} \chi_{[i-1+\varepsilon, i]}\right\|_{E} \leq\left\|\sum_{i \in I} x_{i}\right\|_{E} \leq C_{5} j^{1 / p}
$$


for some constant $C_{5}>0$ which does not depend on $\varepsilon$. Since $E^{\prime \prime}$ has the Fatou property, letting $\varepsilon$ tend to 0 we obtain

$$
C_{4}(1-1 / p)\left\|\sum_{i=1}^{j}(t-i+1)^{-1 / p} \chi_{(i-1, i]}\right\|_{E^{\prime \prime}} \leq C_{5} j^{1 / p} .
$$

Now, since the functions

$$
j^{-1 / p} \sum_{i=1}^{j}(t-i+1)^{-1 / p} \chi_{(i-1, i]} \text { and } t^{-1 / p} \chi_{(0, j]}
$$

are equimeasurable, we have

$$
\left\|t^{-1 / p} \chi_{(0, j]}\right\|_{E^{\prime \prime}} \leq C_{5}\left[C_{4}(1-1 / p)\right]^{-1} .
$$

Using the Fatou property again, we get $t^{-1 / p} \chi_{(0, \infty)} \in E^{\prime \prime}$. Hence $L^{p, \infty} \hookrightarrow E^{\prime \prime}$ and consequently $E^{\prime \prime}=L^{p, \infty}$. Finally, if $E$ is maximal then $E=L^{p, \infty}$, and if $E$ is minimal then $E=L_{0}^{p, \infty}$.

ExAmples. (i) The inclusion $L^{p, \infty} \cap L^{q, \infty} \hookrightarrow L^{1}+L^{\infty}$ is DSS for $1<$ $p \neq q<\infty$.

(ii) If $\varphi$ is a submultiplicative Orlicz function then the inclusion $L^{\varphi} \hookrightarrow$ $L^{1}+L^{\infty}$ is DSS except when $L^{\varphi}=L^{1}$.

REMARK. The submultiplicativity of $\phi_{E}$ is essential in Theorem 4.5: the inclusion operator $L^{p}+L^{q} \hookrightarrow L^{1}+L^{\infty}$ is not DSS for $1 \leq p<q \leq \infty$ (cf. [GHR]).

When considering interpolation spaces obtained by methods of genus $s_{\varrho}$ (e.g. the real and complex interpolation methods) we have the following

COROLlary 4.6. Let $E$ be an r.i. space with submultiplicative fundamental function and $E \neq L^{1}, L^{\infty}, L^{p, \infty}, L_{0}^{p, \infty}$ with $1<p<\infty$. If $F$ is an interpolation space between $E$ and $L^{1}+L^{\infty}$ obtained by an interpolation method of genus $s_{\varrho}$, then the inclusion operator $E \hookrightarrow F$ is DSS.

Proof. This follows from Theorem 4.5 and [GHR, Theorem 3.4].

COROLlary 4.7. Let $E$ be an r.i. space with submultiplicative fundamental function and $E \neq L^{1}, L^{\infty}, L^{p, \infty}, L_{0}^{p, \infty}$ with $1<p<\infty$. If $F$ is an intermediate Banach space between $E$ and $L^{1}+L^{\infty}$ such that

$$
\|x\|_{F} \leq C\|x\|_{E}^{\theta}\|x\|_{L^{1}+L^{\infty}}^{1-\theta}
$$

for some $0<\theta<1$ and $C>0$ and for every $x \in E$, then the inclusion operator $E \hookrightarrow F$ is DSS.

Proof. This follows from Corollary 4.6 and [BL, Theorem 3.5.2(b)].

Finally we give some applications to Lorentz spaces $\Lambda(\phi)$. For classical Lorentz spaces $L^{p, q}$, the inclusion operator $L^{p, q} \hookrightarrow L^{p, q^{\prime}}$ is DSS with $1<$ 
$p<\infty$ and $1 \leq q<q^{\prime} \leq \infty$. This follows easily from Proposition 2.3 and Lemma 2.1 of $\left[\mathrm{CD}_{2}\right]$.

TheOrem 4.8. Let $\phi, \psi \in \Phi$ with $\psi \leq C \phi$ for some constant $C>0$. If

$$
\lim _{t \rightarrow 0, \infty} \frac{\psi(t)}{\phi(t)}=0
$$

and $\phi$ is a submultiplicative function, then the inclusion operator $\Lambda(\phi) \hookrightarrow$ $\Lambda(\psi)$ is $D S S$.

Proof. Suppose that $\Lambda(\phi) \hookrightarrow \Lambda(\psi)$ is not DSS. Then we can find a subspace $S \subset \Lambda(\phi)$ generated by a sequence of disjointly supported functions and $c>0$ such that $\|x\|_{\Lambda(\psi)} \geq c$ for every $x \in S$ with $\|x\|_{\Lambda(\phi)}=1$. By (9), there exists $0<\delta<1$ depending only on $\phi, \psi$ and $c$ such that

$$
\int_{\lambda_{x}(s) \leq \delta} \psi\left(\lambda_{x}(s)\right) d s+\int_{\lambda_{x}(s) \geq 1 / \delta} \psi\left(\lambda_{x}(s)\right) d s \leq \frac{c}{2} .
$$

Hence

$$
\int_{\delta<\lambda_{x}(s)<1 / \delta} \psi\left(\lambda_{x}(s)\right) d s \geq \frac{c}{2}
$$

and therefore

$$
\psi\left(\frac{1}{\delta}\right) \lambda\left\{s \in[0, \infty): \delta<\lambda_{x}(s)<1 / \delta\right\} \geq \frac{c}{2} .
$$

Consequently,

$$
x^{*}(\delta) \geq \frac{c}{2 \psi(1 / \delta)}
$$

This implies that

$$
\|x\|_{L^{1}+L^{\infty}}=\int_{0}^{1} x^{*}(t) d t \geq \int_{0}^{\delta} x^{*}(t) d t \geq \delta x^{*}(\delta) \geq \frac{c \delta}{2 \psi(1 / \delta)} .
$$

This means that the inclusion operator $\Lambda(\phi) \hookrightarrow L^{1}+L^{\infty}$ is not DSS. Now, by Theorem $4.5, \Lambda(\phi)=L^{p, \infty}$ or $\Lambda(\phi)=L_{0}^{p, \infty}$ for some $1<p<\infty$, but this is a contradiction.

REMARK. In general the submultiplicativity of $\phi$ in Theorem 4.8 cannot be removed as the following example shows:

Let $1 \leq r<p<q<s<\infty$. If $\phi(t)=\min \left(t^{1 / p}, t^{1 / q}\right)$ and $\psi(t)=$ $\min \left(t^{1 / r}, t^{1 / s}\right)$ then $\lim _{t \rightarrow 0, \infty} \psi(t) / \phi(t)=0$ and the inclusion operator $\Lambda(\phi)$ $\hookrightarrow \Lambda(\psi)$ is not DSS. Indeed, $t^{-1 / l} \chi_{(0, \infty)} \in \Lambda(\phi)$ for $p<l<q$, so the inclusion $\Lambda(\phi) \hookrightarrow L^{1}+L^{\infty}$, and hence the inclusion $\Lambda(\phi) \hookrightarrow \Lambda(\psi)$, is not DSS.

Acknowledgements. The authors wish to thank the referee for his suggestions and detailed remarks. 


\section{References}

[A] S. V. Astashkin, Disjoint strict singularity of embeddings of symmetric spaces, Mat. Zametki 65 (1999), 3-14 (in Russian); English transl.: Math. Notes 65 (1999), 3-12.

[B] B. Beauzamy, Espaces d'Interpolation Réels: Topologie et Géométrie, Lecture Notes on Math. 666, Springer, 1978.

[BS] C. Bennett and R. Sharpley, Interpolation of Operators, Academic Press, New York, 1988.

[BL] J. Bergh and J. Löfström, Interpolation Spaces. An Introduction, Springer, 1976 .

$\left[\mathrm{CD}_{1}\right] \quad$ N. L. Carothers and S. J. Dilworth, Geometry of Lorentz spaces via interpolation, in: Longhorn Notes, The University of Texas at Austin, Functional Analysis Seminar (1985-6), 107-133.

$\left[\mathrm{CD}_{2}\right] \quad-,-$, Subspaces of $L^{p, q}$, Proc. Amer. Math. Soc. 104 (1988), 537-545.

[CMMM] F. Cobos, A. Manzano, A. Martínez and P. Matos, On interpolation of strictly singular operators, strictly cosingular operators and related operator ideals, Proc. Roy. Soc. Edinburgh, to appear.

[GHR] A. García del Amo, F. L. Hernández and C. Ruiz, Disjointly strictly singular operators and interpolation, ibid. 126 (1996), 1011-1026.

[GHSS] A. García del Amo, F. L. Hernández, V. M. Sánchez and E. M. Semenov, Disjointly strictly-singular inclusions between rearrangement invariant spaces,

J. London Math. Soc. 62 (2000), 239-252.

[HR] F. L. Hernández and B. Rodríguez-Salinas, On $\ell^{p}$-complemented copies in Orlicz spaces II, Israel J. Math. 68 (1989), 27-55.

$\left[\mathrm{K}_{1}\right] \quad$ N. J. Kalton, Orlicz sequence spaces without local convexity, Math. Proc. Cambridge Philos. Soc. 81 (1977), 253-277.

$\left[\mathrm{K}_{2}\right] \quad-$, personal communication, 1996.

[KM] A. Kamińska and M. Mastyło, The Dunford-Pettis property for symmetric spaces, Canad. J. Math. 52 (2000), 789-803.

[KPS] S. G. Kreŭn, Ju. I. Petunin and E. M. Semenov, Interpolation of Linear Operators, Amer. Math. Soc., Providence, 1982.

[K-A] S. A. Kuzin-Aleksinskiu, Weakly compact embeddings of symmetric spaces, Siberian Math. J. 28 (1987), 111-113.

$\left[\mathrm{LT}_{1}\right]$ J. Lindenstrauss and L. Tzafriri, Classical Banach Spaces I, Sequence Spaces, Springer, 1977.

[LT $\left.\mathrm{LT}_{2}\right] \quad-,-$, Classical Banach Spaces II, Function Spaces, Springer, 1979.

$\left[\mathrm{N}_{1}\right] \quad$ S. Ya. Novikov, Boundary spaces for inclusion map between rearrangement invariant spaces, Collect. Math. 44 (1993), 211-215.

$\left[\mathrm{N}_{2}\right] \quad-$, The differences of inclusion map operators between rearrangement invariant spaces on finite and $\sigma$-finite measure spaces, ibid. 48 (1997), 725-732.

[P] E. I. Pustylnik, Some structural properties of the totality of intermediate spaces of a Banach couple, in: Theory of Operators in Function Spaces, Voronezh Gos. Univ., 1983, 80-89 (in Russian).

[PR] D. Przeworska-Rolewicz and S. Rolewicz, Equations in Linear Spaces, Polish Sci. Publ., Warszawa, 1968. 
[W] J. Y. T. Woo, On modular sequence spaces, Studia Math. 48 (1973), 271-289.

Departament of Mathematical Analysis

Faculty of Mathematics

Complutense University

28040 Madrid, Spain

E-mail: pacoh@eucmax.sim.ucm.es

Victor_Sanchez@mat.ucm.es
Department of Mathematics Voronezh State University Voronezh 394693, Russia E-mail: root@func.vsu.ru

Received May 10, 1999

Revised version June 9, 2000 